\title{
Lavado pulmonar bilateral terapéutico en una adolescente con proteinosis alveolar pulmonar. A propósito de un caso
} Therapeutic bilateral lung lavage in an adolescent with pulmonary alveolar proteinosis. Case report

\author{
Dr. Hernán Talamoni ${ }^{a}$, Dr. Néstor Pisapia ${ }^{a}$ Dra. Lorena Cortes ${ }^{a}$, Dra. Marcela Arellano ${ }^{a}$, \\ Dr. Esteban Wainstein ${ }^{b}$ Dra. Graciela Svetliza $a^{b}$ y Lic. Andrea Caneparic
}

\section{RESUMEN}

La proteinosis alveolar pulmonar es una enfermedad pulmonar difusa caracterizada por la acumulación anormal de surfactante y lipoproteínas en el espacio alveolar, lo cual empeora el intercambio gaseoso y lleva a un curso variable desde una presentación clínica asintomática hasta una falla respiratoria grave.

Se presenta a una adolescente de 16 años con este diagnóstico que fue remitida a nuestro Hospital para la realización de un lavado pulmonar total luego de una historia de disnea progresiva, dificultad respiratoria, caída de la función pulmonar y anormalidades radiológicas. Se le realizaron estudios de función pulmonar y mediciones de laboratorio antes y después del lavado pulmonar terapéutico.

Luego, la paciente presentó una inmediata mejoría tanto de los síntomas, radiografía de tórax y mediciones de estudios de función pulmonar como en el intercambio gaseoso. El lavado pulmonar total continúa siendo el estándar de oro para el tratamiento de la proteinosis alveolar pulmonaren casos graves. Palabras clave: proteinosis alveolar pulmonar, lavado pulmonar, insuficiencia respiratoria.

\begin{abstract}
Pulmonary alveolar proteinosis is an unusual diffuse lung disease characterized by abnormal accumulation of pulmonary surfactant and lipoproteins in the alveolar space, which impairs gas exchange with a variable clinical course, ranging from an asymptomatic clinical presentation to severely affected respiratory failure.

A 16-year-old girl with diagnosis of pulmonary alveolar proteinosis presented to our hospital for therapeutic lung lavage after a recent history of progressive dyspnea, respiratory distress, declining lung function measurements, and worsening radiographic abnormalities. We obtained baseline pulmonary function tests and laboratory measurements before and after therapeutic bilateral lung lavage.
\end{abstract}

a. Sección de Neumonología Pediátrica.

b. Sección de Neumonología.

c. Sección de Cuidados Respiratorios Pediátricos, Servicio de Kinesiología.

Hospital Italiano de Buenos Aires, Ciudad de Buenos Aires, Argentina.

Correspondencia:

Dr.Hernán Talamoni: hernan.talamoni@hospitalitaliano.org.ar

Financiamiento: Ninguno.

Conflicto de intereses: Ninguno que declarar.

Recibido: 7-8-2019

Aceptado: 29-10-2019
After the lavage, the patient demonstrated an immediate improvement in symptoms, chest radiograph appearance, pulmonary function test measurements, as well as in gas exchange. Whole lung lavage is still the gold standard for treatment of pulmonary alveolar proteinosis in severe cases. Key words: pulmonary alveolar proteinosis, lung lavage, respiratory insufficiency.

http: / / dx.doi.org/10.5546/ aap.2020.e324

Cómo citar: Talamoni H, Pisapia N, Cortes L, Arellano M, et al. Lavado pulmonar bilateral terapéutico en una adolescente con proteinosis alveolar pulmonar. A propósito de un caso. Arch Argent Pediatr 2020;118(3):e324-e328.

\section{INTRODUCCIÓN}

La proteinosis alveolar pulmonar (PAP) corresponde a una rara enfermedad intersticial difusa que presenta como característica patológica la acumulación de una cantidad excesiva y anormal de material proteináceo rico en lípidos en el interior del espacio alveolar, secundaria al deterioro del procesamiento del surfactante por el macrófago alveolar. ${ }^{1,2} \mathrm{La}$ incidencia se calcula en 0,36 casos por 100000 habitantes con una prevalencia de 3,7 por millón. ${ }^{3}$

Se han descrito 3 formas de presentación clínica:

La forma congénita, que se presenta en los recién nacidos y se asocia a una deficiencia genética de proteínas del surfactante, que, rápidamente, lleva a una insuficiencia respiratoria resistente a todo tipo de terapia. En muchos casos, la única posibilidad terapéutica es el trasplante pulmonar. ${ }^{4,5}$

Una forma secundaria, que se puede asociar a la inhalación de polvos inorgánicos o humos tóxicos, patología neoplásica hematológica (leucemia mieloide aguda, linfomas) o ciertas infecciones (Pneumocystis jirovecii, histoplasmosis, aspergilosis, nocardiosis, virus de la inmunodeficiencia humana -VIH-, etc.). ${ }^{4}$ 
Por último, una forma primaria o autoinmune, que es la más frecuente, pues constituye casi el $90 \%$ de los casos reportados, en la que hay producción de anticuerpos antifactor estimulador de colonias para granulocitos y macrófagos (granulocyte macrophage colony-stimulating factor; GM-CSF, por sus siglas en inglés).4,6

Cualquiera de estas causas produce una alteración funcional o una reducción del número de macrófagos alveolares con la consiguiente acumulación de material proteico a nivel alveolar, que lleva, en los casos más graves, a una alteración en el intercambio gaseoso e hipoxemia. ${ }^{1,7}$

\section{CASO CLÍNICO}

Paciente de 16 años con diagnóstico de PAP que fue remitida a la Sección de Neumonología Pediátrica del Hospital Italiano de Buenos Aires para realizar un lavado pulmonar bilateral terapéutico. Se trató de una adolescente previamente sana, que había comenzado hacía un año con disnea de esfuerzo en forma progresiva y astenia, sin tos ni signos de obstrucción bronquial. No presentaba antecedentes de exposición ambiental u ocupacional.

Fue estudiada en su provincia de origen, donde se constató una radiografía de tórax patológica, y, a partir de allí, se realizaron estudios tomográficos de tórax con imágenes de alta resolución (TCAR), en los que se observó opacidad en vidrio esmerilado, estructuras intralobulillares engrosadas y tabiques interlobulillares con formas típicas poligonales de crazy-paving orientativas de PAP. Por esto, se realizó un lavado broncoalveolar (bronchoalveolar lavage; $B A L$, por sus siglas en inglés), en el que se obtuvo un líquido opaco lechoso, positivo con la tinción de Schiff (periodic acid-Schiff; PAS, por sus siglas en inglés), compatible con esta patología por anatomía patológica. Los cultivos resultaron negativos.
Se decidió derivarla a nuestro Hospital para continuar los estudios y el tratamiento. $\mathrm{Al}$ ingresar, sus signos vitales eran normales, con temperatura de $36,4{ }^{\circ} \mathrm{C}$, presión arterial de $100 / 65 \mathrm{mmHg}$, frecuencia cardíaca de $85 / \mathrm{min}$, frecuencia respiratoria de 23 respiraciones/ minuto y saturación del $93 \%$ en aire ambiente. La exploración física mostró buena entrada de aire bilateral sin ruidos agregados y sin hipocratismo digital, y fue llamativa la disociación entre la escasez de síntomas y hallazgos físicos con las alteraciones radiológicas, de compromiso extenso y bilateral.

En el examen de sangre, hematocrito del $47 \%$ y el resto de la química sanguínea, sin alteraciones. La gasometría arterial en aire ambiente reveló $\mathrm{pH}$ : 7,43; presión parcial de oxígeno $\left(\mathrm{PO}_{2}\right): 74,4 \mathrm{mmHg}$; presión parcial de dióxido de carbono $\left(\mathrm{PCO}_{2}\right): 29,7 \mathrm{mmHg}$; y bicarbonato $\left(\mathrm{HCO}_{3}\right): 21,3 \mathrm{mmHg}$. Estudio reumatológico (anticuerpos anti-ADN de doble cadena -anti-double stranded DNA antibody; anti ds-ADN antibody, por sus siglas en inglés-, anticuerpos anticitoplasma de neutrófilo $\mathrm{P}$ y $\mathrm{C}$ -antineutrophil cytoplasmic antibodies; ANCA P y C, por sus siglas en inglés- y factor antinúcleo -FAN-) dentro de los límites normales y lactato deshidrogenasa (LDH) de 202 UI/1. En la espirometría, se constató incapacidad ventilatoria restrictiva moderada y, en la pletismografía, patrón restrictivo leve.

En el estudio de difusión de monóxido de carbono (DLCO), presentó una disminución con un grado de gravedad de leve a moderado. En la prueba de marcha de 6 minutos, recorrió 280 m y alcanzó una saturación mínima del $87 \%$ al cuarto minuto.

La paciente fue sometida al lavado pulmonar total realizado en dos tiempos. En el primero, se abordó el pulmón izquierdo y, a los 4 días,
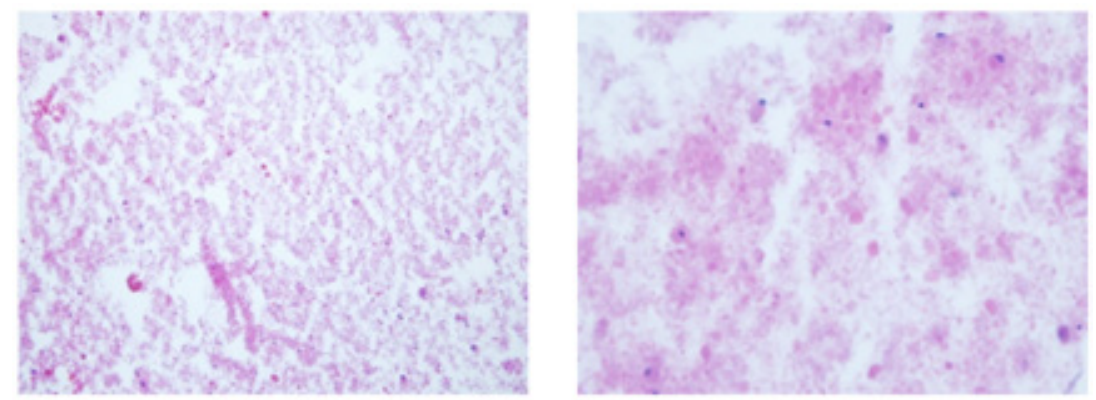

Abundante material proteináceo de fondo, con muy escasas células. 
el pulmón derecho. Se utilizó intubación endobronquial selectiva con tubo doble lumen izquierdo y ventilación selectiva. Se contó con la presencia de un endoscopista respiratorio con experiencia en este procedimiento.

En el primer tiempo quirúrgico (pulmón izquierdo), se introdujo un fibrobroncoscopio pediátrico, se constató la localización del tubo y se realizó un $B A L$, que evidenció material para anatomía patológica y cultivos (Figura 1). Luego se introdujeron alícuotas de 1 litro por vez de solución salina isotónica a $37^{\circ} \mathrm{C}$, que se infundió desde una altura de $40 \mathrm{~cm}$ sobre el paciente. Se realizaron maniobras kinésicas con el objetivo de redistribuir el líquido en el interior del pulmón para luego ser drenado por gravedad. Fue necesario ingresar un total de 14 litros hasta obtener un líquido de egreso totalmente claro. El tiempo de duración del procedimiento en el pulmón izquierdo fue de 2 horas y 20 minutos. Al abordar el pulmón derecho, también se necesitó un total de 14 litros hasta obtener muestras de líquido completamente claro (Figura 2) con una duración de 1 hora y 40 minutos. Los cultivos microbiológicos, incluidos hongos y micobacterias, fueron negativos.

Durante ambos procedimientos y ante el riesgo de una complicación, se contó con la posibilidad de utilizar membrana de oxigenación extracorpórea (extracorporeal membrane oxygenation; ECMO, por sus siglas en inglés). Luego de cada procedimiento, fue llevada a la Unidad de Cuidados Intensivos Pediátricos. Pasó de intubación orotraqueal a ventilación no invasiva durante el mismo día del procedimiento y quedó ventilando espontáneamente a aire ambiente al día siguiente. Ambos procedimientos fueron bien tolerados y sin complicaciones. (a)

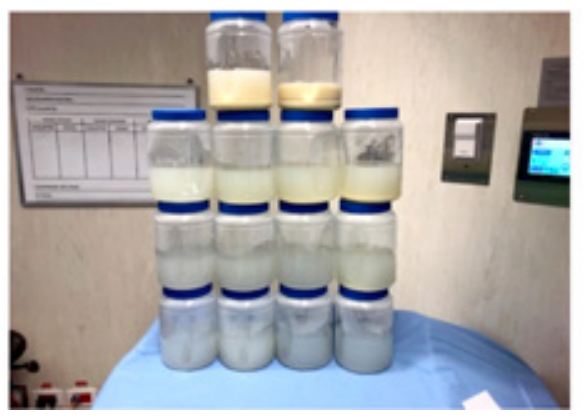

(b)

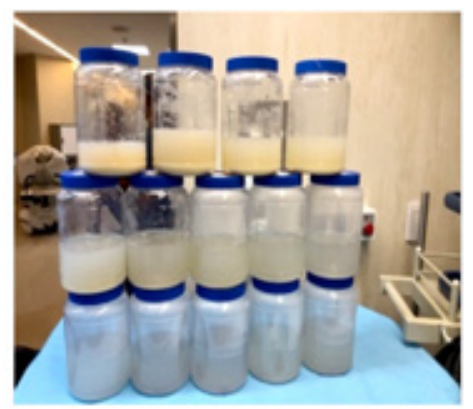

Material obtenido del pulmón izquierdo (a) y del pulmón derecho (b).

FIgURA 3. Estudios tomográficos de tórax con imágenes de alta resolución y radiografías de tórax

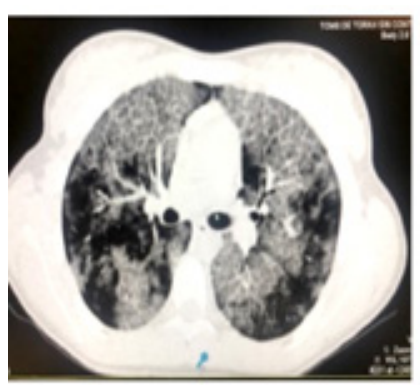

TCAR prelavado

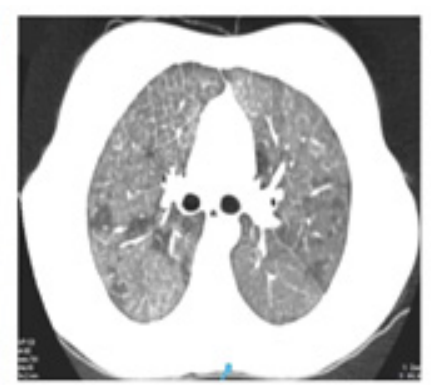

TCAR poslavado

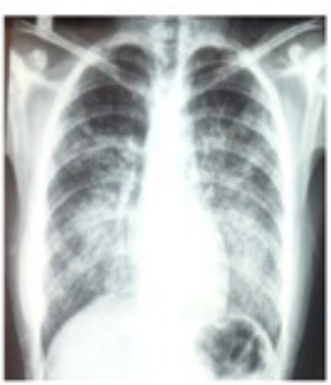

Rx prelavado

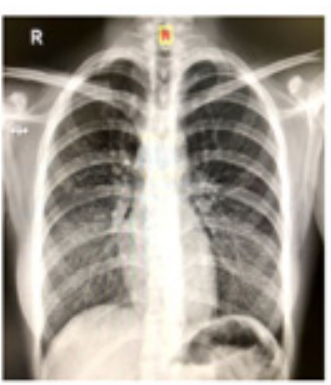

Rx poslavado

TCAR: tomografía de tórax con imagen de alta resolución. Opacidad en vidrio esmerilado, estructuras intralobulillares engrosadas y tabiques interlobulillares con formas típicas poligonales de crazy-paving o empedrado.

Radiografía de tórax: patrón de ocupación alveolar hiliar y perihiliar bilateral y simétrica que se irradia de los hilios hacia la periferia en alas de mariposa. 
Al séptimo día después del procedimiento, se le realizaron estudios de función pulmonar (Tabla 1) e imágenes radiográficas de tórax y TCAR, que presentaron gran mejoría clínica, funcional y radiológica (Figura 3). Se le otorgó el alta hospitalaria al octavo día del lavado pulmonar.

\section{DISCUSIÓN}

El BAL tiene uno de los más altos rendimientos diagnósticos en la PAP, sobre todo, cuando se suma a las imágenes obtenidas con tomografía. La TCAR muestra opacidades en vidrio esmerilado de los espacios alveolares y engrosamiento septal inter- e intralobulillar, conocido como crazy-paving o en empedrado. ${ }^{3,8,9}$

Cuando se realiza el $B A L$, se recupera un fluido turbio o lechoso que contiene un material denso y blanquecino. Su tinción muestra que es paucicelular, con un material de fondo granular basófilo amorfo, $P A S$ positivo. ${ }^{10,11}$ La radiografía de tórax, típicamente, muestra un patrón de ocupación alveolar hiliar y perihiliar bilateral, pero no es patognomónico.

El lavado pulmonar bilateral terapéutico es la forma más efectiva y segura de tratamiento para la PAP. La técnica fue descrita por Ramírez y col. en el año 1963.9,12 Con este procedimiento, se puede obtener una mejoría clínica, fisiológica y radiológica en más del $84 \%$ de los casos después de la primera intervención terapéutica, con un período libre de síntomas que puede ser variable, pero que, en promedio, es de unos $12-14$ meses. $^{13}$ El 30-40\% de los pacientes requiere de un solo lavado. Ante la reaparición de los síntomas, será necesario repetir los lavados pulmonares terapéuticos con cierta regularidad en estos períodos de recaída. ${ }^{13}$

Tabla 1. Análisis de laboratorio pulmonar

\begin{tabular}{lcc}
\hline & Inicial & Tras el BAL \\
\hline FVC (1) & $2,07(59 \%)$ & $2,49(81 \%)$ \\
TLC (l) & $3,24(71 \%)$ & $3,58(79 \%)$ \\
$\% \mathrm{DLCO} \mathrm{ml} / \mathrm{min} / \mathrm{mmHg}$ & $11,38(60 \%)$ & $12,66(67 \%)$ \\
$\mathrm{PaO}_{2}(\mathrm{mmHg})$ & 74 & 94 \\
$\mathrm{~A}-\mathrm{a}$ & 41 & 19 \\
Test de marcha de $6 \mathrm{~m}$ & $280 \mathrm{~m}$, sat. 87\%*480 m, sat. 92 \%* \\
\hline
\end{tabular}

* Menor saturación obtenida durante el estudio.

FVC: capacidad vital forzada.

TLC: capacidad pulmonar total.

DLCO: difusión de monóxido de carbono.

$B A L$ : lavado broncoalveolar.

$\mathrm{PaO}_{2}$ : presión parcial de oxígeno en la sangre arterial.
Campo y col. realizaron un estudio que abarcó 30 centros a nivel mundial donde se realizaron un total de 1110 lavados pulmonares totales por cuadros de PAP tanto en adultos como en niños, durante un período de $18 \pm 11$ años, con un promedio de $5,6 \pm 5$ procedimientos por centro por año. ${ }^{14}$ Las complicaciones más comúnmente reportadas fueron fiebre (el $18 \%$ ), hipoxemia (el $14 \%$ ), sibilancias (el $6 \%$ ) y neumonía (el $5 \%$ ). El derrame pleural y el neumotórax ocurrieron en el 3,1 \% y en el 0,8\%, respectivamente. La mitad de los centros realizaba el lavado en 2 sesiones consecutivas con un intervalo de una a dos semanas. La mitad de los centros se basó en las imágenes para decidir por cuál pulmón comenzar a lavar, mientras que la otra mitad comenzó por el pulmón izquierdo. La posición supina fue elegida por, aproximadamente, el $50 \%$ de los centros. Hubo una gran variabilidad con respecto al volumen instilado por pulmón (entre 5 y 40 litros con un promedio de 15,4 litros/pulmón). ${ }^{14}$ Un efecto adverso preocupante fue la hipoxemia que, generalmente, se revirtió con ventilación en altas concentraciones de oxígeno inspirado, y se debió contar con ECMO en caso de progresar a una falla respiratoria.

Una alternativa al lavado sería la administración del GM-CSF por vía inhalatoria, que es inefectiva en los casos de PAP congénita. Las dosis empleadas y la duración de los tratamientos son variables. ${ }^{15}$

En cualquier caso de PAP, el curso clínico puede ser de tres formas:

1) Estabilidad, pero con síntomas persistentes.

2) Mejoría espontánea o después de la terapia.

3) Deterioro progresivo.

Se presenta un caso de proteinosis alveolar primaria, probablemente, de etiología autoinmune, con un deterioro progresivo de su cuadro clínico, en el que el lavado pulmonar bilateral terapéutico resultó ser una herramienta segura y bien tolerada. Se logró una significativa mejoría clínica, radiológica y funcional en el corto plazo, y quedó la paciente en seguimiento y control por nuestra Sección.

\section{REFERENCIAS}

1. De Arriba C, Antón C, Arreche E, Lorente MP. Proteinosis alveolar pulmonary patrón en empedrado ("crazy-paving") en tomografía computarizada de alta resolución. Anales Sis San Navarrra. 2006; 29(1):127-30.

2. Levin D, Hartman T. Pulmonary alveolar proteinosis. In Hartman T (ed). Pearls Pitfalls in Thoracic Imaging: Variants and Other Difficult Diagnoses. Cambridge: Cambridge University Press; 2011; 50-1.

3. Sánchez-Valadez TI, Carrillo-Muñoz A, Valero-Gómez A, 
Martínez-Pérez S, et al.Proteinosis alveolar. Informe de dos casos y comentario bibliográfico. Neumol Cir Torax. 2015; 74(4):271-5.

4. Cugell DW. Pulmonary alveolar proteinosis. JAMA. 1975; 234(1):80.

5. Nogee LM, De MelloDE, Dehner LP, Colten HR. Deficiency of Pulmonary Surfactant Protein B in Congenital Alveolar Proteinosis. N Engl J Med. 1993; 328(6):406-10.

6. ChanED, King TEJr. Treatment and prognosis of pulmonary alveolar proteinosis in adults. UptoDate. 2017:1-19. [Acceso: 31 de octubre de 2019]. Disponible en: https://www. uptodate.com/contents / treatment-and-prognosis-ofpulmonary-alveolar-proteinosis-in-adults.

7. Boyce DSK, Lee JW, Shah P, Freeman JH, et al. Combinedmodality therapy for pulmonary alveolar proteinosis in a remote setting: a case report. BMC Pulm Med. 2019; 19(1):4-9.

8. Rossi SE, Erasmus JJ, Volpacchio M, Franquet T, et al. "Crazy-paving" pattern at thin-section CT of the lungs: radiologic-pathologic overview. Radiographics. 2003; 23(6):1509-19.
9. GrieseM.Pulmonary AlveolarProteinosis:Acomprehensive Clinical Perspetive Pediatrics. 2017; 140(2):e20170610.

10. Bula GC, Santiago HE, Martínez OB, Reyes M, et al. Proteinosis alveolar. Rev Colomb Neumol. 2017; 29(1):55-62.

11. Goldstein LS, Kavuru MS, Curtis-McCarthy P, Christie HA, et al. Pulmonary alveolar proteinosis: Clinical features and outcomes. Chest. 1998; 114(5):1357-62.

12. Ramírez J, Schultz RB, Dutton RE. Pulmonary alveolar proteinosis: a new technique and rationale for treatment. Arch Intern Med. 1963; 112(3):419-31.

13. Seymour JF, Presneill JJ. Pulmonary alveolar proteinosis: Progress in the first 44 years. Am J Respir Crit Care Med. 2002; 166(2):215-35.

14. Campo I, Luisetti M, Griese M, Trapnell BC, et al. A global survey on whole lung lavage in pulmonary alveolar proteinosis. Chest. 2016; 150(1):251-3.

15. Rodríguez Portal JA. Tratamiento de la proteinosis alveolar primaria del adulto. Arch Bronconeumonol. 2015; 51(7):344-9 